INPLASY

PROTOCOL

To cite: Wang et al. The Role of Carbonic Anhydrase Inhibitors in the Treatment of X-linked Retinoschisis: A Systematic Review and Metaanalysis Meta-analysis. Inplasy protocol 2020120098. doi:

10.37766/inplasy2020.12.0098

Received: 20 December 2020

Published: 20 December 2020

Corresponding author:

Xiaoyan Ding

dingxiaoyan@gzzoc.com

Author Affiliation:

State Key Laboratory of Ophthalmology, Zhongshan Ophthalmic Center, Sun Yat-sen University

Support: NNSF of China (no. 81900896).

Review Stage at time of this submission: Data analysis.

Conflicts of interest: None.

\section{The Role of Carbonic Anhydrase Inhibitors in the Treatment of X-linked Retinoschisis: A Systematic Review and Metaanalysis Meta-analysis}

Wang, R' Li, S2; Liu, Y3; Zhang, X4; Wang, J5; Sun, L6; Zhang, T7; Zhang, $\mathrm{Z}^{8}$; Lin, $\mathrm{H}^{9}$; Ding, $\mathrm{X}^{10}$.

Review question / Objective: What are the changes in vision and retinal thickness in XLRS patients after treatment with carbonic anhydrase inhibitors?

Condition being studied: $\mathrm{X}$-linked retinoschisis (XLRS) is the leading cause of hereditary juvenile-onset macular degeneration in males. It presents with decreased visual acuity and the presence of the inner retina splitting changes on fundus examination. Although the prevalence is not high, ranging from 1 in 15,000 to 1 in 30,000. Vision loss occurs very early in the first decade of life, then progressively to the fifth decade, presents with poor central vision. Furthermore, complications of vitreous hemorrhage and retinal detachment may cause complete vision loss. XLRS is caused by mutations in a gene on the $X$ chromosome called RS1 which encodes a protein involved in intercellular adhesion. Several clinical trials have been conducting to evaluate the efficiency of RS1 gene therapy. To date, there is no specific treatment for XLRS. Carbonic anhydrase inhibitors (CAIs) have been demonstrated to be effective for the improvement of foveoschisis and improvement of vision. There is still very little guidance on the optimal use of CAls. To inform clinical practice and confirm the efficacy and safety, we therefore, conducted a systematic review and meta-analysis of the literature on the efficacy and adverse effects of CAlsl for the treatment of XLRS.

INPLASY registration number: This protocol was registered with the International Platform of Registered Systematic Review and Meta-Analysis Protocols (INPLASY) on 20 December 2020 and was last updated on 20 December 2020 (registration number INPLASY2020120098).

\section{INTRODUCTION}

Review question / Objective: What are the changes in vision and retinal thickness in
XLRS patients after treatment with carbonic anhydrase inhibitors? 
Condition being studied: X-linked retinoschisis (XLRS) is the leading cause of hereditary juvenile-onset macular degeneration in males. It presents with decreased visual acuity and the presence of the inner retina splitting changes on fundus examination. Although the prevalence is not high, ranging from 1 in 15,000 to 1 in 30,000 . Vision loss occurs very early in the first decade of life, then progressively to the fifth decade, presents with poor central vision. Furthermore, complications of vitreous hemorrhage and retinal detachment may cause complete vision loss. XLRS is caused by mutations in a gene on the $X$ chromosome called RS1 which encodes a protein involved in intercellular adhesion. Several clinical trials have been conducting to evaluate the efficiency of RS1 gene therapy. To date, there is no specific treatment for XLRS. Carbonic anhydrase inhibitors (CAIs) have been demonstrated to be effective for the improvement of foveoschisis and improvement of vision. There is still very little guidance on the optimal use of CAls. To inform clinical practice and confirm the efficacy and safety, we therefore, conducted a systematic review and metaanalysis of the literature on the efficacy and adverse effects of CAlsI for the treatment of XLRS.

\section{METHODS}

Search strategy: A.Search strategies for Pubmed - ((Retinoschisin[Title/Abstract]) OR (X-linked retinoschisis[Title/Abstract]) OR (X-linked juvenile retinoschisis[Title/ Abstract]) OR (XLRS[Title/Abstract]) OR (retinoschisis[Title/Abstract]) AND ((Brinzolamide[Title/Abstract]) OR (Dorzolamide[Title/Abstract]) OR (Acetazolamide[Title/Abstract]) OR (carbonic anhydrase inhibitors[Title/ Abstract]) OR (CAls[Title/Abstract]) OR (carbonic anhydrase[Title/Abstract])). B.Search strategies for Embase 1:'retinoschisin'/exp OR retinoschisin OR 'x-linked retinoschisis':ab,ti OR 'x-linked juvenile retinoschisis':ab,ti OR xIrs:ab,ti OR retinoschisis:ab,ti 2:(brinzolamide:ab,ti OR dorzolamide:ab,ti OR acetazolamide:ab,ti OR 'carbonic anhydrase inhibitors':ab,ti OR cais:ab,ti) AND 'carbonic anhydrase':ab,ti 3: 1 AND 2. C.Search strategies for Web of Science - 1: TI=(Brinzolamide OR Dorzolamide OR Acetazolamide OR carbonic anhydrase inhibitors OR CAls OR carbonic anhydrase) $O R A B=(B r i n z o l a m i d e$ OR Dorzolamide OR Acetazolamide OR carbonic anhydrase inhibitors OR CAls OR carbonic anhydrase) 2: $\mathrm{Tl}=($ Retinoschisin OR X-linked retinoschisis OR X-linked juvenile retinoschisis OR XLRS OR retinoschisis) OR $A B=($ Retinoschisin OR $X$ linked retinoschisis $O R \quad X$-linked juvenile retinoschisis OR XLRS OR retinoschisis) 3: 1 AND 2. D.Search strategies for Clinicaltrials.gov and Cochrane Central Register of Controlled Trials. Retinoschisin OR X-linked retinoschisis OR X-linked juvenile retinoschisis OR XLRS OR retinoschisis.

Participant or population: Subjects with Xlinked retinoschisis, and no race and age restrictions.

Intervention: Carbonic anhydrase inhibitors treatment was the main intervention, including oral acetazolamide and topical dorzolamide or brinzolamide.

Comparator: Compare the outcomes before and after CAls treatment: Changes in visual acuity and central foveal zone thickness on optical coherence tomography during follow-up for the duration of treatment.

Study designs to be included: Observational studies, Case Series $(n>3)$, Randomized trials.

Eligibility criteria: 1 . STROBE score greater than 20. 2. The visual acuity or macular thickness data before and after treatment are documented. The visual acuity data can be converted to logMAR. The macular thickness is measured by OCT and recorded by um. 3. The number of case reports must be greater than 3. 4. Papers that can be translated into English.

Information sources: The information extracted from electronic databases and 
trial registers, including the PubMed, OVID Embase databases, Web of Science, Cochrane Central Register of Controlled Trials, and CIinicalTrials.gov (www.clinicaltrials.gov). In studies where only figures or graphs were presented, the images were analyzed with the WebPlotDigitzer program (http:// arohatgi.info/ WebPlotDigitizer/, accessed November 29, 2015) to obtain numerical values.

Main outcome(s): Best-corrected visual acuity (BCVA) and the central macular thickness (CMT) are the most important outcome for the evaluation of the therapeutic interventions of XLRS patients. All included papers have documented at least one of them. The BCVA data were converted into log Mar, and all the CMT parameters were measured by OCT and recorded in um. Data at baseline and at each time point after treatment were reextracted and analyzed.

Quality assessment / Risk of bias analysis: A scoring system based on the STROBE checklist was developed. The total score is 26 points. Each item on the score sheet was evaluated as being complete ( 1 point), partial ( 0.5 points), incomplete (0 points), or not applicable. Studies with scores greater than $\mathbf{2 0}$ were included. The risk of bias was assessed by two independent reviewers using the Cochrane Risk of Bias tool.

Strategy of data synthesis: Due to the inconsistent observation time of different papers, when evaluating the intervention effects of CAls, we selected the latest posttreatment time point of each individual for the meta-analysis, also the subgroup analyses of different CAls (acetazolamide, brinzolamide, dorzolamide) was conducted. In addition, in order to analyze the time effect, we extracted the data and divided it into 4 groups independently. The results measured at 1 month, 2 months, or 3 months after treatment are classified as group A. Similarly, Group B: 4M, 5M, 6M, Group C: 11M, 12 M, 13 M, and Group D: $\geq 14$ M. RevMan 5.4.1 software was accessed to conduct the meta-analysis.
The mean difference (MD) and $95 \% \mathrm{Cl}$ were calculated for statistical analyses, as the results of BCVA and OCT were continuous data. The heterogeneity of the effect size across the studies was tested using the $\mathbf{Q}$ statistic (P50\% was considered heterogeneous). If there was significant heterogeneity between studies, a randomeffects model was used; otherwise, a fixedeffects model was used.

Subgroup analysis: 1. Effecticy of different CAls (acetazolamide, brinzolamide, dorzolamide) 2. Outcomes at different follow-up periods (Group A, Group B, Group C, Group D), versus baseline data were conducted

Sensibility analysis: 1.Change inclusion criteria (especially controversial studies) exclude low-quality studies; 2. Alter the statistical model (for example applying a fixed-effects model in the sensitivity analysis where a random-effects model has been used initially and vice versa).

\section{Country(ies) involved: China.}

Keywords: Carbonic anhydrase inhibitors; $\mathrm{X}$-linked juvenile retinoschisis; Congenital retinal disease; Meta analysis.

Contributions of each author:

Author 1 - Ruixin Wang.

Email: ruiruiw413@aliyun.com

Author 2 - Songshan li.

Author 3 - Yafen Liu.

Author 4 - Xiayin Zhang.

Author 5 - Jinhui Wang.

Author 6 - Limei Sun.

Author 7 - Ting Zhang.

Author 8 - Zhaotian Zhang.

Author 9 - Haotian Lin.

Email: gddlht@aliyun.com

Author 10 - Xiaoyan Ding.

Email: dingxiaoyan@gzzoc.com 